\title{
WAVELET APPLICATION IN COMPRESSION OF A REMOTE SENSED IMAGE
}

\author{
Balasubramanian. R \\ Research Scholar - Remote Sensing \\ Systems Operator, Mechanical Engineering, CEGC \\ Anna University, Chennai, INDIA 600025 \\ balu.varagur@annauniv.edu
}

\author{
Ramakrishnan. S. S. \\ Professor of Remote Sensing \\ Inst. of Remote Sensing, Civil Engg., CEGC \\ Anna University, Chennai, INDIA 600025 \\ drssramakrishnan@yahoo.com
}

\begin{abstract}
Present scenario gives importance to precision image representing information about remote sensing, medicine, seismic etc. which are more importance to decision makers and researchers. These precision data are of very high resolution in nature in order to give optimal information which in turn occupies higher level of disk space. The main practical problem in transmitting these information are that primarily it requires a higher bandwidth, greater time involved in reception etc. There are numerous data compression algorithms but the primary concern is that the compression should be Lossless before and after reception. This paper employs the mathematical tool 'Wavelet' for the precision data compression and decompression. The advantage of compression permits the decision maker to process and send back after modifications, in the base image data. Wavelets find a greater application in obtaining lossless compression technique. Wavelet decomposes the data into various frequency components that helps to study and analyze each component with a resolution matched to its scale.
\end{abstract}

Key Word : Wavelet, Bandwidth, Classifier, Lossless, Lossy, Quantized

\section{INTRODUCTION}

This work is an attempt to study the quality of the classified image after compressing the original remote sensing data acquired by the sensor. The need for compression is to achieve an optimal usage of disk space, high speed of transmission in the networks, optimal time of data downloading, effective usage of band width and modulation techniques. The compressed and decompressed image has more complete lossless information.

The objectives of the paper are:

to classify a image obtained from a remote sensed satellite sensor

to check the size of the classified image

to compress the classified image using Wavelet techniques

to check the size of the compressed image

to decompress the image

to check the size of the decompressed image display the obtained image to check for quality and any loss of information. Ideally data compression should not distort the spectral characteristics and retain the basic colour content. This is more important for calibration, geo-referencing, ensuring the targets that are spectrally separable in the original data. In addition Wavelet compression also filters noise and ambiguity.

\section{Study area}

The study area chosen is the Chennai metropolitan area. This area lies between $80^{\circ} 11^{\prime} 3^{\prime \prime}$ to $80^{\circ} 13^{\prime} 26^{\prime \prime}$ latitude and $13^{\circ}$ $17^{\prime} 33^{\prime \prime}$ to $13^{\circ} 5^{\prime} 24^{\prime \prime}$ longitude. The extent of the geographical area is covered by Survey of India (SOI) topographic maps 66 $\mathrm{C} / 4$ and $\mathrm{C} / 8$. This area is covered by False Colour Composite (FCC) Path-Row 102-64 geo-coded. The study area is characterized by the presence of major features such as roads, railway lines, urban with vegetation, urban without vegetation, water bodies, forest areas and stadiums. The remote sensed satellite image of the study area is shown in Fig. 1.

\section{Image compression}

Compression is used to reduce the size of digital data. There are two types of compressions: Loseless and Lossy.

\section{Loseless}

In a lossless compression algorithm, compress data can be used to recreate an exact replica of the original. No information is lost to the compression process. This type of compression is also known as entropy coding. This name comes from the fact that a compressed signal is generally more random than the original. Patterns are removed when a signal is compressed. This compression is useful for exact reconstruction.

\section{Lossy}

In this compression the original data cannot be exactly reconstructed from the compressed data. The reason is that much of the detail in an image can be discarded without greatly changing the appearance of the image. Here none of the fine 
details are included, but the most important aspects of the image are retained.

\section{Compression Methodology}

For compression the basis is Transform coders that are based upon mathematical transforms. Then the data is reprocessed. An invertible transform is performed on the data so that the coefficients are decimated (quantized) and the quantized coefficients are entropy encoded. The objective is to transform the image and then set to zero those values which do not greatly influence the signal.

\section{Wavelet Transform}

Wavelets are signals which are local in time and generally have an irregular shape. The name wavelet comes from the fact that they integrate to zero; they wave up and down across the axis. Many wavelets also display a properly ideal for compact signal representation orthogonally. This property ensures that data is not over represented. A signal can be decomposed into many shifted and scaled representation of the original mother wavelet. A wavelet transform can be used to decompose a signal in to components wavelets. Once this is done the coefficients of the wavelets can be decimated to remove some of the details. Wavelets have the great advantage of being able to separate the fine details in a signal. Very small wavelets can be used to isolate very fine details in a signal, while very large wavelets can identify coarse details. In addition, there are many different wavelets to choose from.

One particular wavelet may generate a more sparse representation of a signal than another, so different kinds of wavelets must be examined to see which is most suited to image compression. The discrete wavelet transform already exists. This transform decomposes a given signal into shifted and scaled versions of a single 'mother' wavelet as in Equation 1. The transform itself is lossless.

$$
\psi_{s}, \tau(t)=\mathrm{s}^{-1 / 2}\left[\psi \frac{\lfloor t-\tau]}{s}\right]
$$

\section{Wavelet properties}

The most important properties of wavelets are the admissibility and the regularity conditions and these are the properties which gives wavelets their name. It can be shown that square integral functions $\infty(\mathrm{t})$ satisfying the admissibility condition as given in Equation 2.

$$
\int\left[|\psi(\omega)|^{2} /|\omega|\right] \mathrm{d} \omega<\infty
$$

This is used to first analyze and then reconstruct a signal without loss of information. The $\psi(t)$ stands for the Fourier transform of $\psi(\mathrm{t})$. The admissibility condition implies that the Fourier transform of $\psi(\mathrm{t})$ vanishes at the zero frequency illustrated in Equation 3.

$$
|\psi(\omega)|_{\omega=0}^{2}=0
$$

This means that wavelets must have a band pass like spectrum. This is a very important observation, which is used to build an efficient wavelet transform. Zero value at the zero frequency means that the average value of the wavelet in the time domain must be zero as in Equation 4. Thus

$$
\int \psi(\mathrm{t}) \text { at }=0
$$

Therefore it must be oscillatory. In other words, $\psi(\mathrm{t})$ should be a wave.

As can be seen from Equation 2, the wavelet transform of one-dimensional function is two-dimensional. The wavelet transform of a two-dimensional function is four-dimensional. The time-bandwidth product of the wavelet transform is the square of the input signal and for most practical applications this is not a desirable property. Therefore one imposes some additional conditions on the wavelet functions in order to make the wavelet transform decrease quickly with decreasing scales. These are the regularity conditions and they state that the wavelet function should have some smoothness and concentration in both time and frequency domains. Regularity is a quite complex concept and it may be explained using the concept of vanishing moments.

If expanded the wavelet transform equation (5.1) into the Taylor series at $\mathrm{t}=0$ until order $\mathrm{n}$ (let $\tau=0$ for simplicity) we get the series as in the Equation 5 .

$$
\gamma(\mathrm{s}, 0)=1 / \sqrt{\mathrm{s}}\left[\Sigma \mathrm{f}^{(\mathrm{p})}(0) \mathrm{I}\left[\mathrm{t}^{\mathrm{p}} / \mathrm{p} !\right] \psi(\mathrm{t} / \mathrm{s}) \mathrm{dt}+\mathrm{O}(\mathrm{n}+1)\right]
$$

Here $\mathrm{f}^{(\mathrm{p})}$ stands for the $\mathrm{p}^{\text {th }}$ derivative of $\mathrm{f}$ and $\mathrm{O}(\mathrm{n}+1)$ means the rest of the expansion. Now, if we define moments of the wavelet by $M_{p}$, Equation 6 is obtained

$$
M_{p}=\int t^{p} \psi(t) d t
$$

then we can rewrite into the finite development as in Equation 7

$$
\begin{aligned}
\gamma(\mathrm{s}, 0)= & (1 / \sqrt{\mathrm{s}})\left[\mathrm{f}(0) \mathrm{M}_{0} \mathrm{~s}+\left(\mathrm{f}^{(1)} / 1 !\right) \mathrm{M}_{1} \mathrm{~s}^{2}+\left(\mathrm{f}^{(2)} / 2 !\right) \mathrm{M}_{2} \mathrm{~s}^{3}\right. \\
& \left.\left.+\ldots \ldots \ldots+\left(\mathrm{f}^{(\mathrm{n})} / \mathrm{n} !\right)^{\mathrm{n}+1}\right)\right]
\end{aligned}
$$

From the admissibility condition it is observed that the $0^{\text {th }}$ moment $\mathrm{M}_{\mathrm{o}}=0$, so that the first term in the high hand side of Equation 7 is zero. If we are to make the other moments up to $M_{n}=0$ as well, then the wavelet transform coefficients $(s, 0)$ will decay as fast as $s^{n+2}$ for a smooth signal $f(t)$. This is known in scientific parlance as the vanishing moments Equation 2 or approximation order. If a wavelet has $\mathrm{N}$ vanishing moments, then the approximation order of the wavelets transform is also $\mathrm{N}$. The moments do not have exactly zero, a small value is often good enough. In fact, experimental research suggests that the number of vanishing moments required depends heavily on the application. 
To summarize, the admissibility condition results the wave, regularity and vanishing moments results in the fast decay or the let. Thus when combined, they results in Wavelet.

\section{RESULTS AND DISCUSSIONS}

The study area image was rectified and preprocessed. Then compression technique was employed. Both the compressed and uncompressed images were imported to the image processing software for marking training set samples for various classes like beach, vegetation, water bodies, urban with and without vegetation as in Fig. 2. The training set selection of various features is shown in Fig. 3. Maximum likelihood technique is adopted for classification. The classified outputs of the uncompressed and compressed images are shown in Fig. 4 \& 5 respectively.

\section{CONCLUSIONS}

Can achieve excellent compression

The classified output of the uncompressed and compressed images resembles the same so that it is lossless

Wavelet techniques can be employed for compression to save the bandwidth requirements for data transmission of precision images

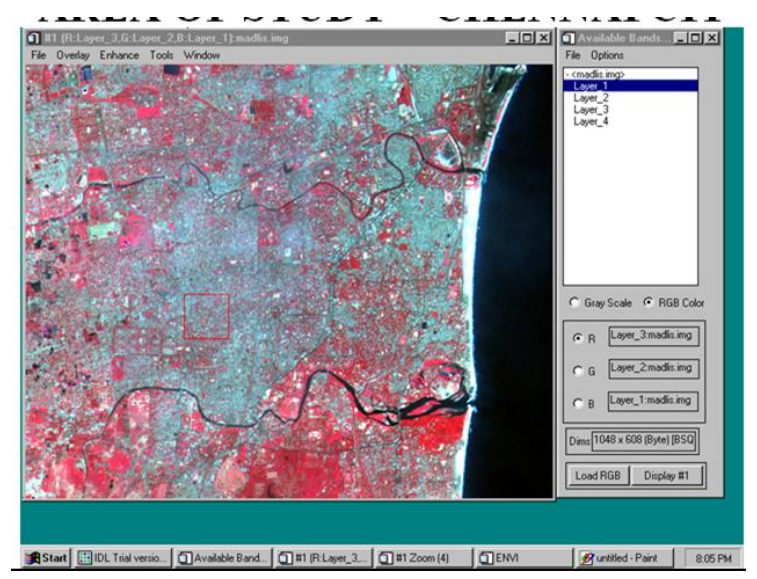

Fig. 1. Study Area - Chennai Metropolitan Area

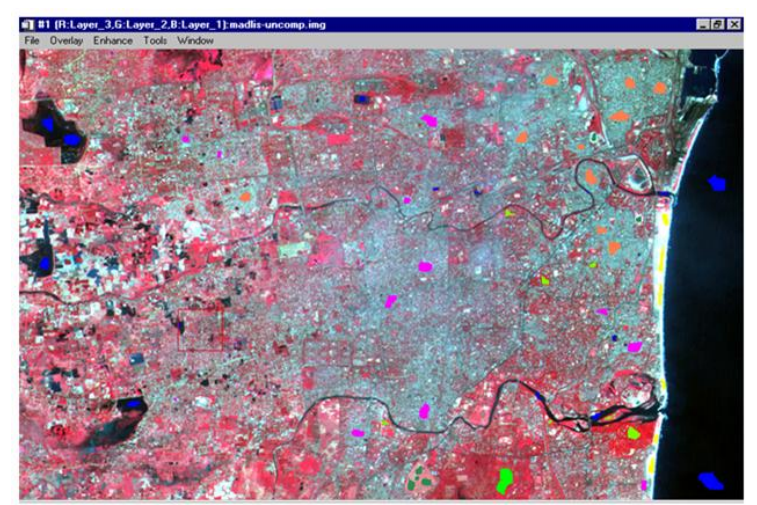

Fig. 2. Training set

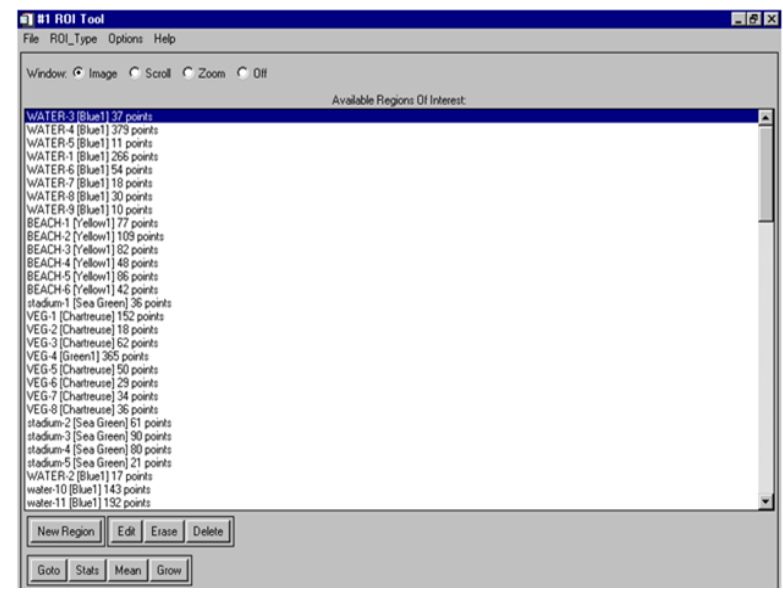

Fig. 3 Training set features

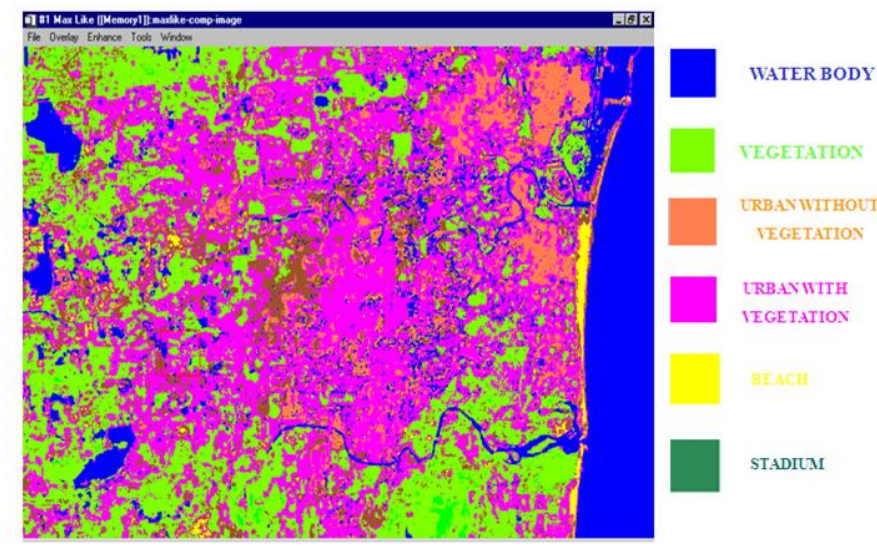

Fig. 4 Classified output of Compressed image

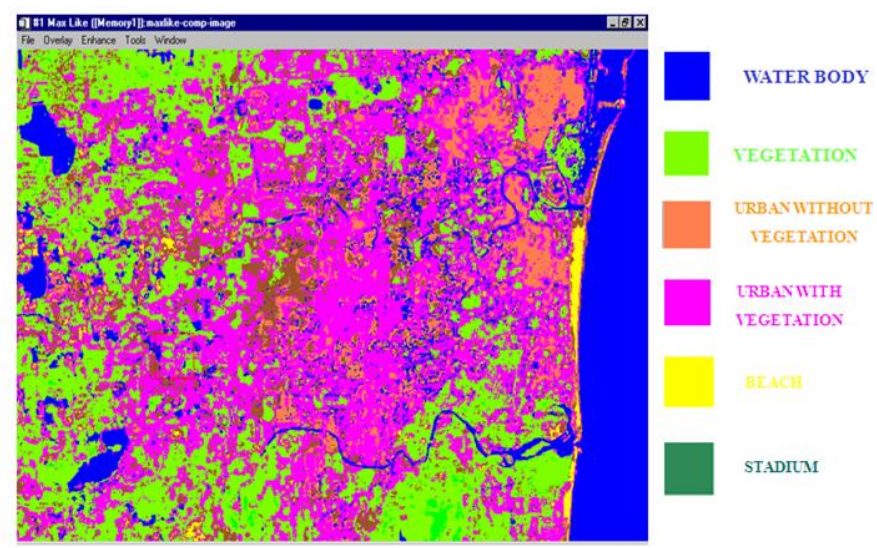

Fig. 5 Classified output of Uncompressed image

\section{ACKNOWLEDGMENT}

Sincere thanks to Anna University and Prof. Dr. L. Karunamoorthy, HoD, Mechanical Engg and Prof. Dr. Murugesan, Professor, Remote Sensing, Anna University, Chennai-25 for their encouragement \& support and M/s Exelis Visual Information Systems for providing the ENVI software. 


\section{REFERENCES}

[1] A. S. Lewis and G. Knowles, "Image Compression using 2D wavelet Transformation", IEEE Trans, IP, vol. 1, no. 2, 1992, pp. 244-250.

[2] Floyd F. Sabins, "Remote Sensing Principles and Interpretation", W. H. Freeman and Company, New York, 1996.

[3] W. Staurt and Nixon, "Compressed Image Quality versus Compressed Image Size", 1998 (http://www.wavelet.org/compressionwhitepaper1.pdf)

[4] Lakshman Prasad, and S. S. Iyengar, "Wavelets Applications to Image Processing", Academic Press, Boston, 1999.

[5] Hong Pan, Li-Zuo Jin, Xiao-Hui Yuan, Si-Yu Xia and Liang-Zheng Xia, "Content based Embedded Image Compression using Binary wavelet Transform", Image and Vision computing, vol. 28, issue.6, June 2010, pp. 991-1002. 